\section{A Possible Galactic Origin for Quasi-stellar Sources}

The interpretation of the observed red-shifts of "quasars" as cosmological gives rise to severe difficulties concerning both the energy output and the variation of light because these objects have then to be placed at enormous distances.

We wish to suggest the possibility that the observed red-shifts result from an inflow of matter at relativistic velocities in the gravitational field of a highly condensed white dwarf with a very large escape velocity. To establish a starting point, consider a collapsed body with mass $10^{33} \mathrm{~g}$ and a radius of several kilometres; these stars must exist ${ }^{1}$ and will strongly attract interstellar matter.

The high escape velocity - a large fraction of the velocity of light-implies that nothing will escape apart from photons and neutrinos, thus there can be no net flux of matter and thus no stellar wind. If the star is a member of a binary system there will be an outward flux of matter owing to the stellar wind of the companion, but if the condensed star is isolated it must accrete interstellar matter.

The accretion process should be quite efficient. If the interstellar medium has an ambient magnetic field accreted material will bring its own field lines with it which become trapped in the star. Also the star will possess in its wake a concentrated magnetic field directed so as to guide material into the star. If the interstellar gas has a uniform motion over distances of the order of $G M / v^{2}$, where $v$ is the relative velocity of gas and star, then under the influence of gravitation all matter will concentrate in the wake which becomes a region of high density, temperature and magnetic field. The star accretes material at the rate (capture cross-section times velocity) $G^{2} M^{2} / v^{3} \rho$, where $\rho$ is the ambient density of the interstellar material. Taking $M=10^{33} \mathrm{~g}, v=5 \times 10^{5} \mathrm{~cm} / \mathrm{sec}$, and $\rho=10^{-24} \mathrm{gm} / \mathrm{c} . c$., the accretion rate is $10^{11} \mathrm{~g} / \mathrm{sec}$. With an escape velocity of $\frac{1}{2} C$ the accreted material supplies energy to the object at a rate $10^{31} \mathrm{ergs} / \mathrm{sec}$. If this energy is radiated as blackbody radiation the effective temperature of the star would be a few hundred thousand degrees. Thus this star would have a luminosity one hundredth of that of the Sun. Moreover, at a distance of 100 parsecs it would have a magnitude of about $15^{\mathrm{m}}$, which is within the observed range of quasar apparent magnitudes; the parallax of such a star $0.01^{\prime \prime}$ would be very difficult to observe directly.

Material moving into the wake experiences a much greater magnetic field and incoming electrons will gain energy by the betatron effect and would be expected to produce large amounts of synchrotron radiation. (Such radiation would be expected to have a maximum intensity where the material first approaches the wake because here the energy associated with transverse velocity components would be dissipated. Thus we might expect a strong source of radio emission at a distance $G M / v^{2}$. With the values already given this would be at $10^{2}$ astronomical units from the star.) Furthermore, the optical spectrum would be produced very near the stellar surface where densities and pressures are high so that the radiation would be strongly red-shifted by almost the amount of the escape velocity; the considerable gravitational redshift of the star itself would be added to this.

In summary, a single highly condensed star interacting with the interstellar medium should give rise to the following effects: (1) A double radio source, the two members of which would be separated by about $G M / v^{2}$. (2) Associated with one radio source is a point source with a continuum characteristic of a very hot body with superposed very broad strongly red-shifted emission lines. The red-shifts observed depend on the orientation of the star with respect to the Sun. (3) Optical radiation of the synchrotron type is associated with the other radio source. (4) Both sources should be time-variable because the density, velocity and magnetic field of the interstellar field is dependent on time. (5) The optical point source should produce soft X-rays.

The sensitive parameter is $v$, the relative velocity of star and interstellar medium, because the accretion rate is proportional to $v^{-3}$ and the source separation to $v^{-2}$.

\section{G. J. ODGERS}

Dominion Astrophysical Observatory, R.R. 7, Victoria, British Columbia.

\section{R. W. Stewart}

Department of Physics,

University of British Columbia,

Vancouver.

${ }^{1}$ Schwarzschild, M., Structure and Evolution of the Stars, 232 (Princeton University Press, 1958).

\section{PLANETARY SCIENCE}

\section{Comparisons of Frequency by Long Path Very Low Frequency Transmissions}

Most of the reliable measurements of the frequency stability of very low frequency (VLF) propagation paths have been made over the North Atlantic path, and over paths of the same order of length. Pierce et al. ${ }^{1}$ used atomichrons on each side of the Atlantic and found a standard deviation of 2 in $10^{11}$ for frequency comparisons consisting of $24 \mathrm{~h}$ averages for the $G B R$ (Rugby, $16.0 \mathrm{kc} / \mathrm{s}$ ) transmission over a path length of $5,200 \mathrm{~km}$. It has been suggested by Watt and Plush ${ }^{2}$ that the standard deviation of phase - and thus of frequency over a constant interval of time-would be proportional to the square root of path length. However, Chilton et al. ${ }^{3}$ give figures suggesting that it may be proportional to the square of the path length squared.

Although the results presented here are not sufficient to resolve this question, it has been found that over very long paths there is little increase in the standard deviation above Pierce's value. Over the $G B R-$ New Zealand path of $18,700 \mathrm{~km}$ the $24 \mathrm{~h}$ standard deviation of frequency is 5 in $10^{11}$, and for the $W W V L$ (Colorado, $20 \cdot 0 \mathrm{kc} / \mathrm{s}$ )-New Zealand path of $12,200 \mathrm{~km}$ the corresponding figure is 3 in $10^{11}$. These figures were obtained by intercomparisons between the received signals from both stations and a rubidium gas cell frequency standard. It has not been possible to determine to what extent they were affected by receiver phase shifts and they therefore represent an upper limit to the uncertainty due to purely propagation effects. The observations were made in December 1965; at this time of the year the anomalous phase effect observed at times over the $G B R$-New Zealand path does not occur ${ }^{4}$. National Physical Laboratory corrections were, of course, applied to the $G B R$ figures, but the National Bureau of Standards corrections to $W W V L$ are negligible for the present purpose.

It should be noted that the GBR path crosses the auroral zone and is, therefore, likely to be somewhat more disturbed than the $W W V L$ path, even in the geomagnetically quiet conditions which prevailed during December 1965. The figures, therefore, give tentative support to the suggestion of Watt and Plush that a square root proportionality is in operation. Their main importance, however, lies in showing that, at least during geomagnetically quiet periods, VLF frequency comparisons over a $24 \mathrm{~h}$ period should, under normal conditions, be well within \pm 1 in $10^{10}$ over the longest path lengths.

\section{B. GERARD}

Physics and Engineering Laboratory,

Lower Hutt, New Zealand.

${ }^{1}$ Pierce, J. A., Winkler, G. M. R., and Corke, R. L., Nature, 187, 914 (1960). 'Watt, A. D., and Plush, R. W., J. Re8. Nat. Bur. Stand., 68D, 35 (1959). Chilton, C. J., Crombie, D. D., and Jean, A. G., Propagation of Radio Waves at Frequencies below $300 \mathrm{~K} c / 8,257$ (Pergamon Press, Oxford, 1964). - Gerard, V. B., J. Atmos. Terrest. Phys., 28, 425 (1966). 